DOI: https://doi.org/10.46296/gt.v4i8edesp.0030

\title{
PERFIL FAMILIAR EN PACIENTES CON TRATAMIENTO DE HEMODIÁLISIS
}

\section{FAMILY PROFILE IN PATIENTS WITH HEMODIALYSIS TREATMENT}

\author{
López-Palma Jessica Alexandra ${ }^{1}$; Ortiz-Zambrano Jhon Elvin ${ }^{2}$ \\ ${ }^{1}$ Estudiante de la Maestría Académica con Trayectoria de Investigación en Psicología, Mención \\ Psicoterapia. Instituto de Posgrado de la Universidad Técnica de Manabí, UTM. Portoviejo, \\ Ecuador. Correo: jlopez4980@utm.edu.ec. ORCID ID: https://orcid.org/0000-0002-8207-5748 \\ ${ }^{2}$ Docente Investigador de la Universidad Técnica de Manabí, UTM. Portoviejo, Ecuador. \\ Correo: jhon.ortiz@utm.edu.ec. ORCID ID: https://orcid.org/0000-0002-3533-5084
}

\begin{abstract}
Resumen
El entorno familiar experimenta cambios sobre la estructura y su funcionamiento en la readaptación del paciente al nuevo estilo de vida, por las alteraciones que se generan en la enfermedad renal y el tratamiento de hemodiálisis. Objetivo: evaluar la funcionalidad familiar y caracterizar la tipología familiar que poseen las familias de los pacientes con tratamiento de hemodiálisis. Materiales y Método: es un estudio descriptivo, de corte transversal, con una muestra de 21 pacientes que cumplían con los criterios de inclusión. Se emplearon un cuestionario y la Escala de FACE $3^{\circ}$ versión. Resultados: el funcionamiento familiar que predominó en las familias fue el rango medio con $52,4 \%$; la balanceada un $38,1 \%$ y las extremas con $9,5 \%$. La familia nuclear con $47,6 \%$ prevaleció como el tipo familiar. Se encontró diferencias significativas mínimas con respecto a la tipología familiar y a los niveles de funcionamiento familiar. Conclusión: los pacientes con IRCT perciben el funcionamiento familiar con disfuncionalidad moderada, son familias que se encuentran vinculadas a los miembros de manera relacionada, ante la poca incapacidad de cambios muestran inflexibilidad y rigidez que se suscitan dentro del sistema familiar en el cuidado del paciente en TRS que no permite una funcionalidad adecuada.
\end{abstract}

Palabras clave: Funcionalidad familiar, cuidadores, hemodiálisis.

\begin{abstract}
The family environment undergoes changes on the structure and its functioning in the readadtation of the patient in the new lifestyle, due to the alterations generated in kidney disease and in hemodialysis treatment. Objective: To evaluate family functionality and characterize the family typology possessed by the families of patients undergoing hemodialysis treatment. Materials and method: it is a descriptive cross-sectional study with a sample of 21 patients who met the inclusión criteria. A questionnaire and the 3rd versión FACE scale were used. Results: The family functioning that predominated in the families was the medium range with $52.4 \%$, the balanced $38.1 \%$ and the extreme ones with $9.5 \%$. The nuclear family with $47.6 \%$ prevailed as the family type Minimal significant differences were found with respect to family typology and levels of family functioning. Conclusion: Patients with IRCT perceive family functioning with moderate dysfunction, they are families that are linked to the members in a related way, given the little inability to change they show inflexibility and rigidity that arise within the family system in patient care in TRS that does not allow adequate functionaliy.
\end{abstract}

Keywords: family functionality; caregivers; hemodialysis.

Información del manuscrito:

Fecha de recepción: 13 de julio de 2021.

Fecha de aceptación: 27 de septiembre de 2021.

Fecha de publicación: 12 de noviembre de 2021. 


\section{Introducción}

La Insuficiencia Renal Crónica Terminal (IRCT) describe la pérdida global e irreversible de la función renal. La evolución gradual de la enfermedad hace necesario la implementación de un Tratamiento Renal Sustitutivo (TRS) de diálisis o hemodiálisis, o de trasplante renal. De acuerdo con el registro del Ministerio de Salud Pública del Ecuador (2018) el aumento creciente anual en la prevalencia de pacientes en tratamiento por diálisis es del $8 \%$. Se estima que existen cerca de diez mil personas en tratamiento con hemodiálisis y diálisis peritoneal, que representa una tasa de 660 casos por millón de habitantes, siendo la hemodiálisis el TRS que más prevalece.

Se considera la cuarta causa de mortalidad general y la quinta de mortalidad prematura. La mortalidad alcanza niveles entre el $6 \%$ y $7 \%$. La IRCT constituye la patología más frecuente y la que mayor costo genera dentro de los programas de salud pública. En la actualidad la IRCT y la necesidad de TRS es una problemática que presenta un sinfín de personas a nivel mundial, resulta esencial conocer el impacto y la repercusión que se genera a corto y largo plazo en la evolución de la enfermedad y el tratamiento.

La terapia renal sustitutiva es un tratamiento que implica cambios importantes en la vida del enfermo, deberá adaptarse a los tiempos que conlleva realizar los intercambios dentro del tratamiento dialítico (Jiménez et al., 2018). La hemodiálisis es un procedimiento demandante e invasivo, que ejerce las funciones vitales del sistema de filtración de la sangre, eliminando los residuos tóxicos e inadecuados para el organismo (Costa et al., 2016).

Las limitaciones impuestas por el tratamiento de hemodiálisis se perciben como el deterioro continuo y constante de varios años, provocando la necesidad de readaptación de la nueva condición que no solo repercute en la vida del paciente, también en el aspecto familiar por la falta de reconocimiento de los cambios. Rescova (2011) destaca que las intervenciones dirigidas a la prevención, el retraso de la progresión, mejorar la calidad de vida y el bienestar del paciente son de gran importancia. En ese 
bienestar resulta fundamental incluir a la familia.

Actualmente, la familia es uno de los contextos más relevante en la vida del ser humano, desempeña un papel clave en las experiencias vividas, en el proceso de saludenfermedad y el desarrollo del individuo al constituir la más importante y efectiva red de apoyo social (Trevín et al., 2016). Las familias son sistemas que funcionan de forma eficaz en diferentes culturas, opera a través de patrones transaccionales que se dan entre sus miembros, las mismas que son mantenidas en el tiempo (Minuchin \& Fichman, 2004).

La familia cumple funciones protectoras y reguladoras en los procesos y cambios que atraviesan los miembros dentro del sistema familiar. $\quad \mathrm{Al}$ existir resquebrajamientos del sistema familiar puede perder su identidad e integridad. Gamboa \& Acosta (2014) define el funcionamiento familiar como el sistema que integra a todos los procesos psicológicos que ocurren en el seno de la familia y permite un adecuado desarrollo como grupo de los individuos que la conforman.
El funcionamiento familiar está relacionado con la estructura familiar, la interacción de vínculos afectivos percibidos por los miembros de la familia, la capacidad de cambio, el grado de flexibilidad del sistema familiar, que proporciona el intercambio de homeostasis 0 disfuncionalidad de la familia. Para que una familia pueda ser considera funcional o no, es necesaria evaluarla, puede ser estudiada en términos de su estructura, o por la forma como está organizada en un momento dado, y por sus procesos los cuales cambian (Sigüenza, 2015).

Las investigaciones han demostrado que la aparición de una enfermedad terminal en alguno de los miembros de la familia representa un serio problema en su funcionamiento como en su composición (Onque, 2016). Estructuralmente existe una modificación sobre las jerarquías, límites, alianzas y coaliciones establecidas por la familia entre sus miembros a causa de que el enfermo adquiere la centralidad familiar (Torres et al., 2018).

Pérez, (2020) en sus tesis "Perfil familiar del paciente con enfermedad renal crónica con terapia de diálisis 
peritoneal" determinó el perfil familiar del paciente con enfermedad renal crónica en terapia de diálisis peritoneal en el HGR1 IMSS Querétaro. Mediante un estudio descriptivo, transversal y prolectivo, encontró predominio en el tipo de familia nuclear simple en el $50 \%$, en la funcionalidad familiar un rango medio del $48.9 \%$. La familia con diálisis peritoneal perteneció al tipo nuclear simple con un adecuado funcionamiento familiar ante la enfermedad.

Martínez et al., (2017) en el estudio sobre "Funcionamiento familiar en pacientes con insuficiencia renal crónica terminal tratados con hemodiálisis" analizaron el funcionamiento familiar en pacientes en hemodiálisis del Hospital Naval de Especialidades de Veracruz, concluyó que las familias con disfunción moderada predominó, la tipología familiar que prevaleció fue las familias nucleares con disfunción grave y hubo una prevalencia de familias aglutinadas y caóticas en relación a la cohesión y la adaptabilidad con la disfunción familiar grave.

Trevín et al., (2016) en un estudio descriptivo para caracterizar las familias de pacientes con IRCT que fueron ingresados en la sala de nefrología del Hospital Clínico Quirúrgico Celia Sánchez Manduley en el año 2014. Estableció predominio de las familias funcionales, señalando que la dimensión más afectada fue la permeabilidad y la presencia de criticidad severa en las familias, resaltando que las dificultades de las familias entorpecen muchas veces el trabajo de las instituciones y servicios de salud.

En Ecuador en el 2013, en las ciudades de Quito y Riobamba en un estudio relacional entre la Funcionalidad familiar y estado de salud en pacientes con insuficiencia renal crónica en tratamiento con hemodiálisis, de la clínica de los riñones Menydial. Determinó que las dos ciudades presentan familias funcionales, perciben una buena funcionalidad familiar al igual que los pacientes, se encontró familias moderadamente funcionales $y$ disfuncional con niveles bajo en ambas ciudades, no se encontraron correlaciones entre funcionalidad familiar y los aspectos que mide el estado de salud (Jordán \& Mora, 2013). 
La familia y su funcionamiento no se puede generalizar, al existir diversos tipos de familias, su funcionamiento variará por múltiples factores como agentes externos e internos de la familia (Sigüenza, 2015). Es posible asegurar que el conocimiento de una familia es un elemento ineludible para el entendimiento y comprensión del individuo, su conducta y actuación (Garibay, 2013).

El propósito del estudio es establecer el funcionamiento familiar como recurso de apoyo fundamental en la recuperación y readaptación del paciente dentro del proceso de la enfermedad renal y del tratamiento de hemodiálisis. Ante el poco abordaje que presenta la variable a estudiar, ya que las investigaciones se han limitado a tratar temas entorno a la salud del paciente, dejando de lado el funcionamiento familiar.

Para ello se enfatiza en el funcionamiento familiar $y$ en la tipología familiar que poseen las familias de los pacientes con tratamiento de hemodiálisis dentro del mantenimiento o abandono de los síntomas que demanda la enfermedad y en cómo repercute entre sí. A través del estudio se pueda contribuir a una base de datos que beneficie al proceso de atención familiar dentro del tratamiento renal enfocado hacia el paciente y la familia de manera integral.

\section{Materiales y métodos}

\subsection{Diseño}

Es un estudio cuantitativo, transversal, de tipo descriptivo, en pacientes con Insuficiencia renal crónica terminal en tratamiento de hemodiálisis en la Clínica Municipal de Diálisis Montecristi (CMDM).

\subsection{Método de selección}

La selección fue no probabilística y se determinó bajo los criterios de selección, con una muestra inicial de 30 pacientes que asisten a tratamiento de hemodiálisis, la muestra final fue de 21 pacientes que cumplían los criterios de inclusión.

\subsection{Criterios de inclusión, exclusión y eliminación}

Fueron incluidos los pacientes que reciben tratamiento hemodialítico en la CMDM, convivan con un familiar, estén dispuestos a participar en el estudio y aceptaron firmar el consentimiento informado. Se 
excluyó a los pacientes que no convivan con un familiar, no autorizaron su participación, no accedieron de forma voluntaria a firmar el consentimiento informado y desertaron del proceso de investigación. Se eliminó a los pacientes que fueron transferidos a otra unidad renal y fallecieron.

\subsection{Técnicas de recolección de información}

Los instrumentos que se aplicaron en el desarrollo de la investigación fueron un cuestionario dirigido a los pacientes para determinar los datos de afiliación, la tipología familiar y la temporalidad del tratamiento en hemodiálisis, el instrumento fue validado por la autora y el tutor de la investigación.

Para evaluar el funcionamiento familiar se utilizó la Escala de Cohesión y Adaptabilidad Familiar $3^{\circ}$ versión (FACES III), creada por Olsen, Portner y Lavee 1985; adaptada en el año 2005 a la población española por Martínez, con un nivel de fiabilidad de 0,89 en Cohesión y 0,87 en Adaptabilidad. Consta de 20 ítems, 10 evalúan la cohesión que son pares y 10 la adaptabilidad que son nones mediante una escala Likert del 1 al 5.
Según la puntuación obtenida la funcionalidad se clasifica en tres categorías: familias funcionales (balanceada), familias con disfunción moderada (rango medio) y familias con disfunción grave (extremas) (Schmidt et al., 2010). Se empleó esta adaptación del instrumento y se llevó a cabo por el investigador la aplicación durante el tratamiento de hemodiálisis.

\subsection{Procedimientos de datos}

En la generación de datos se manejó el programa Statistical Package for the Social Sciences (SPSS) versión 25, mediante los resultados obtenidos se realizó un análisis descriptivo de las variables (datos de afiliación, tipología familiar, temporalidad del tratamiento de hemodiálisis y funcionalidad familiar) a través del método estadístico de frecuencia.

Para el análisis de las tablas cruzadas se relaciona la funcionalidad familiar con las variables de cohesión, adaptabilidad, tipología familiar y la presencia de discapacidad, los resultados se digitaron en tablas de frecuencia absoluta y porcentual. 


\subsection{Consideraciones éticas}

La información obtenida en la investigación es confidencial y la revelación de la información se dará bajo las condiciones establecidas en el consentimiento informado y se garantiza el uso de los datos con fines de investigación. Se declara no hay conflicto de intereses

\section{Análisis de resultados}

El estudio se realizó con 21 pacientes que están en tratamiento de hemodiálisis, el $76,2 \%$ de la población son del sexo masculino y un $23,8 \%$ son femenino, la edad promedio es de 51 a 61 años con un $47,6 \%$. La presencia de discapacidad es de $28,6 \%$; el $19 \%$ representa discapacidad sensorial y el 9,5\% discapacidad física, el 71,4\% se encuentran en tratamiento de hemodiálisis entre un año y 5 años.

La funcionalidad familiar se determinó en 2 dimensiones, con el $47,6 \%$ el grado de cohesión corresponde al nivel intermedio relacionada, los pacientes perciben a la familia con una buena unión familiar que permite mantener la estructura familiar de manera adecuada en el proceso de enfermedad y tratamiento. En el grado de adaptabilidad el $33.3 \%$ sitúa a las familias flexibles en un nivel intermedio, los miembros de la familia como los pacientes se adaptan a los cambios propios que conlleva la enfermedad renal y el proceso de hemodiálisis con flexibilidad lo cual permite la homeóstasis en el sistema familiar.

En la tabla 1, las familias de rango medio predominó con el $52,4 \%$ la alteración en una de la dimensión (cohesión o adaptabilidad familiar) dificulta el funcionamiento, según los resultados la adaptabilidad familiar presenta un porcentaje alto en el nivel de rigidez dentro del sistema familiar como en el tratamiento renal, al estar determinados los roles de los miembros, las reglas y las relaciones, dificultad la posibilidad de cambio entre el paciente y la familia impidiendo un correcto funcionamiento familiar. En las familias balanceadas prevaleció con el $38,1 \%$ las dimensiones de cohesión y de adaptabilidad son centrales, los resultados indican que a pesar de las reestructuraciones que se han dado en el sistema familiar dentro del TRS el paciente y la familia mantienen la funcionalidad 
entre los miembros. Las familias extremas se ubican en los extremos con un $9,5 \%$ siendo las dimensiones de cohesión y la adaptabilidad disfuncionales tanto en el paciente como en el sistema familiar al estar establecidas la composición y estructura familiar.

Tabla 1. Funcionalidad Familiar en los pacientes en tratamiento de hemodiálisis.

\begin{tabular}{ccc|c|c|c}
\hline & & Frecuencia & Porcentaje & $\begin{array}{c}\text { Porcentaje } \\
\text { válido }\end{array}$ & $\begin{array}{c}\text { Porcentaje } \\
\text { acumulado }\end{array}$ \\
\hline \multirow{3}{*}{ Válido } & Balanceada & 8 & 38,1 & 38,1 & 38,1 \\
\cline { 2 - 6 } & Rango medio & 11 & 52,4 & 52,4 & 90,5 \\
\cline { 2 - 6 } & Extrema & 2 & 9,5 & 9,5 & 100,0 \\
\cline { 2 - 6 } & Total & 21 & 100,0 & 100,0 & \\
\hline
\end{tabular}

El nivel de funcionamiento familiar $y$ el grado de cohesión familiar establece el $28,57 \%$ a las familias balanceadas de tipo relacionada en un nivel intermedio, los pacientes en TRS perciben los límites generacionales claros entre los miembros de la familia, establecen alianza, comparten espacios, tiempos e intereses sin perder la individualidad el paciente y el sistema familiar. El $19,5 \%$ representa a las familias de rango medio con niveles de cohesión bajo e intermedio que corresponde a familias no relacionada $y$ relacionada. Al ser familias con disfunción moderada la interacción que presenta el paciente dentro de la familia es poco e influye los múltiples factores externos e internos que se subsistan dentro de la enfermedad renal y del tratamiento de hemodiálisis en el hogar. En las familias extremas el porcentaje es de $4,76 \%$ la unión familiar es la no relacionada y la aglutinada, ambos niveles de cohesión se encuentran en los extremos bajo y alto, de acuerdo con los resultados la funcionalidad familiar incidió en la unión familiar situando como rígidos a los límites generacionales y con extrema separación emocional a la familia.

En el nivel de funcionamiento familiar y en el grado de adaptabilidad con un $23,81 \%$ representa las familias balanceadas con el grado de flexibilidad en un nivel intermedio, los pacientes con IRCT percibe los cambios que se presentan dentro del TRS y en el sistema familiar como flexibles en los aspectos de poder, 
asertividad, roles y en las reglas. Las familias con rango medio muestran rigidez en un nivel bajo, con un $23,57 \%$ la estructura familiar muestra poca unión familiar entre los miembros y el liderazgo autoritario imposibilita la opción de cambio en los roles y las reglas dentro del funcionamiento familiar. Las familias extremas son rígida y caótica, se vincula con un $4,76 \%$ en niveles bajo y alto donde ambos se ubican en los extremos, la ambivalencia del control parental y el autoritarismo en los pacientes no permite a la familia estabilidad de cambio y una adaptación adecuada en el proceso de enfermedad- tratamiento.

La tipología familiar que predominó en los pacientes con IRCT en tratamiento de hemodiálisis es la familia nuclear con $47,6 \%$ donde el entorno familiar convencional se ha mantenido ante las crisis que ha atravesado la familia entorno a la enfermedad renal y a los cambios que se han generaron dentro del sistema familiar en la readaptación del nuevo estilo de vida del paciente y la familia, sin alterar la tipología familiar. Las puntuaciones son en base al parentesco de la familia.

En la tabla 2, se encontró diferencias significativas mínimas con respecto a la tipología familiar y a los niveles de funcionamiento familiar, la familia nuclear fue el contexto familiar que predominó con una funcionalidad de rango medio de un $28,6 \%$ siendo las familias convencionales las que puntúan una escasa cohesión y adaptabilidad de los recursos familiares dentro del proceso de la IRCT y del tratamiento de hemodiálisis. La familia monoparental presentó una funcionalidad de rango medio de $19 \%$ al estar determinada la estructura familiar con un solo miembro en la red familiar los pacientes en TRS no perciben el apoyo necesario que demanda la enfermedad. En la familia extensa la funcionalidad que prevaleció fue la balanceada con $14,3 \%$ los pacientes perciben el funcionamiento y la organización familiar que se presenta en la IRCT y en el proceso dialítico de forma adecuada. 
Tabla 2. Composición de la tipología familiar y el funcionamiento familiar de los pacientes en tratamiento de hemodiálisis.

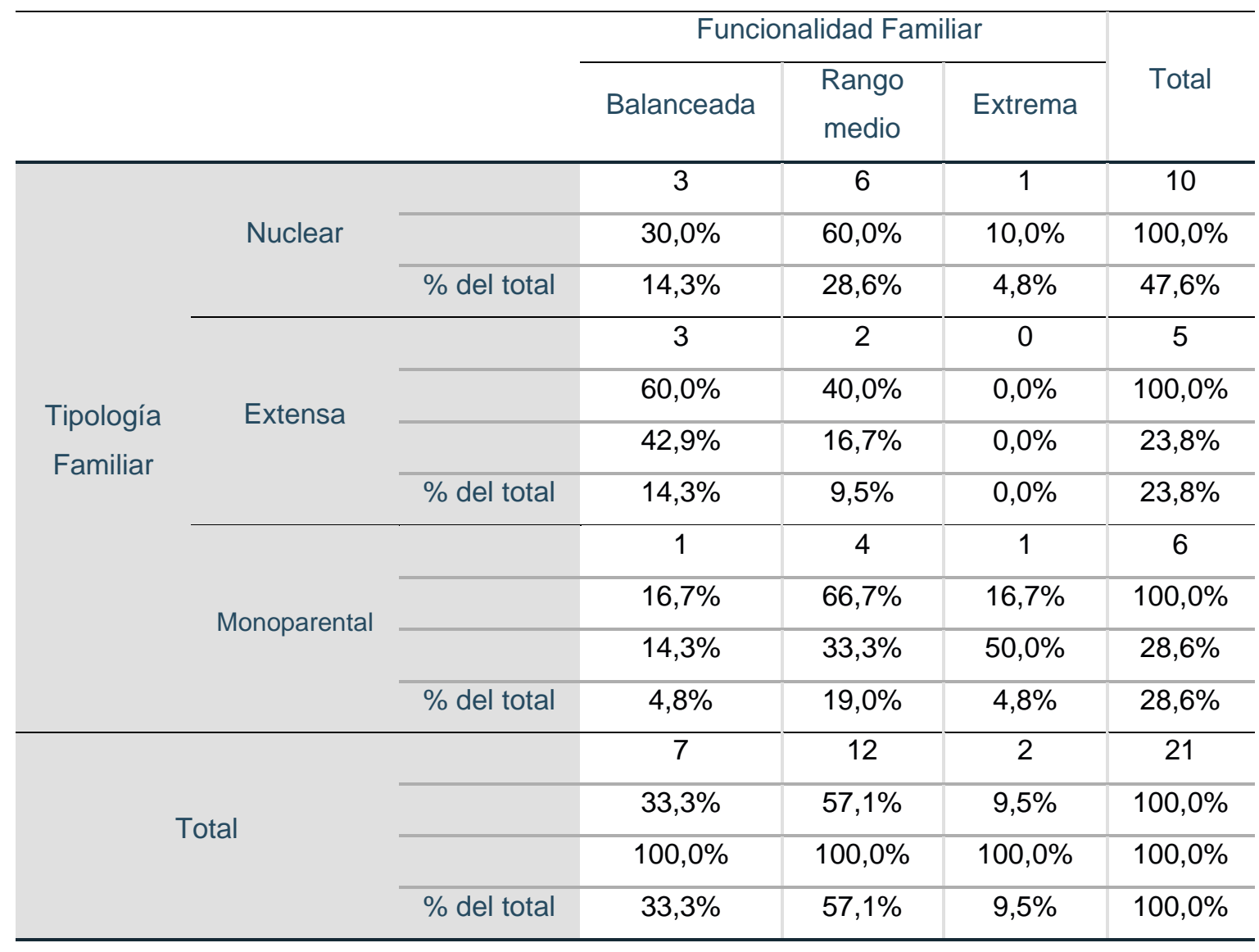

El nivel del funcionamiento de rango medio predominó en dos tipologías familiares por lo tanto se observó que todas las estructuras familiares presentaron cambios dentro del funcionamiento, en efecto el tipo de familia no influyó en la funcionalidad o disfuncionalidad en el hogar.

La presencia de discapacidad física y sensorial con relación a los niveles de la funcionalidad familiar mostraron el funcionamiento de rango medio en las 2 discapacidades con una puntuación de $66,7 \%$ la sensorial y el 33,3\% la física. Las limitaciones de la IRCT y del tratamiento ocasionadas por el deterioro propio de la enfermedad renal, acarrea a los pacientes a padecer algún tipo de discapacidad y ubica a la familia como la red de apoyo fundamental, al no tener una adecuada funcionalidad genera disfunción moderada por las altas demandas de atención y supervisión. 


\section{Discusión}

En los pacientes con IRTC en tratamiento de hemodiálisis se encontró que el sexo que más índice mostró fue el masculino, los grupos de edades corresponden a la etapa de adultez y vejez, en comparación con el estudio de (Martinez et al., 2017) la población que predominó fue el sexo femenino y los rangos de las edades se presentaron desde etapas más tempranas, aumentando la incidencia de la enfermedad renal en el avance de la salud.

Dentro de la tipología familiar, la familia nuclear sigue siendo la ideal $y$ forma parte fundamental dentro del proceso de enfermedad-tratamiento. Los reportes de diferente estudio presentaron similares resultados con la estructura familiar, los pacientes en su mayoría son familias nucleares que responde a diferentes patrones culturales de la sociedad, es importante el apoyo que ofrece la familia nuclear en las etapas de crisis que se subsistan.

El impacto ocasionado por la presencia de algún tipo de discapacidad dentro del TRS es una variable poco abordada, se observó que los pacientes ante la pérdida o disminución de la autonomía personal cambian todos los esquemas, la familia ante el poco reconocimiento de los sus recursos adopta medidas de afrontamiento inadecuados que se vuelven rígidas a los cambios. En el estudio no se determina las dimensiones que componen el funcionamiento familiar y como esta se altera ante la presencia de discapacidad, lo cual resulta fundamental investigar en otros estudios y poder conocer las características que representa la familia como sistema.

Los recursos familiares y redes de apoyo que prevaleció en los pacientes con TRS fue el funcionamiento familiar de rango medio o disfunción moderada, tanto para el paciente y la familia la IRCT acarrea diversos cambios en la calidad de vida y en el sistema familiar que en ocasiones llegan a ser drástico, las dificultades que atraviesa la familia pueden afectar en el grado de cohesión 0 de adaptabilidad familiar e imposibilitar el cambio en el funcionamiento familiar de forma apropiada. Los resultados obtenidos de la investigación de Pérez, (2020) presentan similitud con los resultados alcanzados. En relación 
con el estudio de Azcarate et al., (2006) que predominó el funcionamiento familiar funcional, donde estableció al equipo de salud como red de apoyo en el cuidado del paciente y la reorganización de tareas y funciones que involucra al paciente y su familia. Resultados que no se presentaron en el estudio.

\section{Conclusiones}

La mayoría de los pacientes con IRCT perciben el funcionamiento familiar con disfuncionalidad moderada, son familias que se encuentran vinculadas a los miembros de manera relacionada, ante la poca incapacidad de cambios muestran inflexibilidad y rigidez que se suscitan dentro del sistema familiar en el cuidado del paciente en TRS que no permite una funcionalidad adecuada.

El núcleo familiar es parte esencial dentro del tratamiento dialítico, la tipología familiar que prevaleció en el estudio fueron las familias nucleares, sin importar el tipo de familia se evidenció la presencia de disfunción moderada en la estructura, donde el grado de cohesión y adaptabilidad de los miembros influirá en cómo se dé la relación entre estas dos dimensiones.

El deterioro que genera la propia enfermedad renal conlleva a la presencia de discapacidad en los pacientes a corto o largo plazo, la funcionalidad familiar se ve afectada por la alta demanda de atención y supervisión del paciente. En los resultados del estudio la disfuncionalidad familiar se presentó en ambas discapacidad sensorial o físicas donde se evidencia la asociación entre la presencia de discapacidad con el funcionamiento familiar.

\section{Bibliografía}

Azcarate, E., Ocampo, P., \& Quiroz, J. (2006). Funcionamiento familiar en pacientes integrados a un programa de diálisis peritoneal: Intermitente y ambulatoria. Archivos en medicina familiar, 8(2). Recuperado de: https://www.medigraphic.com /pdfs/medfam/amf2006/amf062f.pdf

Costa, G., Pinheiro, M., Medeiros, S., Costa, R., \& Cossi, M. (2016). Calidad de vida en pacientes con insuficiencia renal crónica en hemodiálisis. Enfermería Global, 15(43), 59-73, ISSN: 1695-6141. Recuperado de: 
https://scielo.isciii.es/pdf/eg/v 15n43/clinica3.pdf

Gamboa, P. (2014). "El apoyo familiar y la calidad de vida de los pacientes con insuficiencia renal crónica terminal atendidos en el hospital IESS de Ambato. Repositorio de la Universidad Técnica de Ambato, 28-31. Recuperado de:

https://repositorio.uta.edu.ec/ bitstream/123456789/7860/1/ FJCS-POSG\%20040.pdf

Garibay, S. (2013). Enfoque sistémico, una introducción a la terapia familiar sistémica. ISBN: $\quad$ 978-607-448-339-0. México: Editorial El Manual Moderno S.A, 2. Recuperado de:

https://www.academia.edu/37 729816/Enfoque_sist\%C3\%A 9mico_una_introducci\%C3\% B3n_a_la_terapia_familiar_si st\%C3\%A9mica_Salvador_G aribay_Rivas

Jiménez, Y., \& Carrillo, G. (2018). "Reencontrándome a través de la diálisis peritoneal": Un abordaje fenomenológico. Enfermería Nefrológica, 21(3), 275-283, ISSN: 22542884. Recuperado de: https://doi.org/10.4321/s225428842018000300010

Jordán, M., \& Mora, Z. (2013). Funcionalidad familiar $y$ estado de salud en pacientes con insuficiencia renal crónica en tratamiento con hemodiálisis, de la clínica de los riñones Menydial de las ciudades de Quito y Riobamba en los meses de junio a julio de 2013. Pontificia Universidad Católica del Ecuador. Recuperado de: http://repositorio.puce.edu.ec/ bitstream/handle/22000/5927/ T-PUCE6082.pdf?sequence $=1$ \&isAllo wed $=\mathrm{y}$

Martínez, R., Islas, F., \& Nájera, A. (2017). Funcionamiento familiar en pacientes con insuficiencia renal crónica terminal tratados con hemodiálisis. Revista Mexicana de medicina familiar, 4 (26)30. Recuperado de: http://www.revmexmedicinafa miliar.org/files/rmf_2017_1_0 26-030.pdf

Ministerio de Salud Pública del Ecuador. (2018). Prevención, diagnóstico y tratamiento de la enfermedad renal crónica. Dirección Nacional de Normalización-MSP.

Recuperado de: http://salud.gob.ec

Minuchin, S., \& Fichman, V. (2004). Familias y terapia familiar. ISBN: $\quad$ 968-852-041-1. Barcelona, España: Editorial Gedisa, S.A. Recuperado de: https://www.academia.edu/23 701962/Minuchin_S_1979_F amilias_y_Terapia_Familiar_ Barcelona_Gedisa 
Onque, P. (2016). Factores socioeconómicos $y$ funcionalidad familiar en pacientes con insuficiencia renal crónica terminal de la unidad de diálisis peritonealESSALUD TACNA 2016. Universidad Nacional del Altiplano Puno, 1, 1-94. Recuperado de: http://repositorio.unap.edu.pe/ handle/UNAP/3258

Pérez, B. (2020). Perfil familiar del paciente con enfermedad renal crónica con terapia de diálisis peritoneal. Universidad Autónoma de Querétaro. Recuperado de: http://ring.uaq.mx/bitstream/1234567 89/2037/1/MEESC-2669100220-328-

Berenice\%20P\%c3\%a9rez\% 20Calder\%c3\%b3n\%20\%20A.pdf

Rescova, E. (2011). Caracterización emocional de los cuidadores de pacientes en tratamiento renal sustitutivo por hemodiálisis. Universidad Central «Marta Abreu» de las Villas. Recuperado de: https://dspace.uclv.edu.cu/bit stream/handle/123456789/20 88/Estefania\%20Rescova.pdf ?sequence $=1$ \&isAllowed $=y$

Schmidt, V., Barreyro, J., \& Maglio, A. (2010). Escala de evaluación del funcionamiento familiar FACES III: ¿Modelo de dos o tres factores? Escritos de
Psicología, 3(2), 30-36, ISSN: 1138-2635, 1989-3809.

Recuperado de: https://www.redalyc.org/articu lo.oa?id=271019811003

Sigüenza, W. (2015). "Funcionamiento familiar según el modelo Circumplejo de Olson". Universidad de Cuenca, 24. Recuperado de: https://dspace.ucuenca.edu.e c/bitstream/123456789/21878 /1/TESIS.pdf

Torres, X., Sánchez, J., \& Estrella, J. (2018). Funcionalidad familiar y enfermedad renal diabética. Revista de Medicina, 22(2), 67-72, ISSN: 1390-8464. Recuperado de: https://doi.org/10.23878/medi cina.v22i2.877

Trevín, G. M., Vargas, S. R., \& Vázquez, B. Á. (2016). Funcionamiento familiar en pacientes con insuficiencia renal crónica en tratamiento dialítico. MULTIMED, 20(1), 75-89, ISSN: 1028-4818. Recuperado de: https://www.medigraphic.com $/ \mathrm{pdfs} / \mathrm{multimed} / \mathrm{mul}$ 2016/mul161g.pdf 